\title{
Efficacy and Safety of 8- or 12 Weeks of Glecaprevir/ Pibrentasvir in Patients with Evidence of Portal Hypertension
}

\author{
Robert S. Brown Jr. (D) - Michelle A. Collins - Simone I. Strasser • \\ Amanda Emmett · Andrew S. Topp · Margaret Burroughs · \\ Rosa Ferreira · Jordan J. Feld
}

Received: December 1, 2021 / Accepted: January 28, 2022 / Published online: February 17, 2022

(C) The Author(s) 2022

\begin{abstract}
Introduction: High efficacy and safety of 8-week glecaprevir/pibrentasvir (G/P) therapy was seen in hepatitis C (HCV)-infected, treatment-naïve (TN), compensated cirrhosis (CC) patients in EXPEDITION-8. To provide further understanding of the efficacy of G/P treatment in $\mathrm{HCV}$-infected $\mathrm{TN}$ patients with $\mathrm{CC}$ and clinical evidence of portal hypertension (PHT), this analysis focused on differences in sustained virologic response at post-treatment week 12 (SVR12) between 8-week and 12-week G/P treatment groups in patients with PHT, and on differences in safety outcomes between PHT and non-PHT groups.
\end{abstract}

R. S. Brown Jr. ( $ه)$

Center for Liver Disease and Transplantation, Weill Cornell Medical College, 1305 York Avenue, New York, NY 10021, USA

e-mail: rsb2005@med.cornell.edu

M. A. Collins · A. Emmett · A. S. Topp ·

M. Burroughs · R. Ferreira

AbbVie Inc., North Chicago, IL, USA

S. I. Strasser

AW Morrow Gastroenterology and Liver Centre, Royal Prince Alfred Hospital, Sydney, NSW, Australia

\section{J. J. Feld}

Toronto Centre for Liver Disease, University Health Network, University of Toronto, Toronto, ON, Canada
Methods: Data were derived from an ad hoc subgroup analysis of the EXPEDITION-8 study for patients receiving 8 weeks of G/P therapy, and pooled patient-level data from nine clinical studies for patients receiving 12 weeks of therapy. Evidence of PHT included at least one of the following at baseline: FibroScan $\geq 20 \mathrm{kPa}$, platelets $<100 \times 10^{9} / \mathrm{L}$, or medical history consistent with PHT. The primary efficacy endpoint was SVR12; adverse events (AEs) consistent with hepatic decompensation were assessed.

Results: PHT was identified in 60.6\% (208/343) and $57.1 \%(224 / 392)$ of the 8 - and 12-week groups, respectively. For those with PHT, SVR12 was $97.6 \%$ $(203 / 208)$ and $98.7 \%(221 / 224)$ with 8- and 12-week treatment, respectively (intention-to-treat population). For those without PHT, 97.8\% (132/ $135)$ in the 8-week group and $97.6 \%(164 / 168)$ in the 12-week group achieved SVR12. Eight patients with PHT, and seven without, did not achieve SVR12. Similar rates of AEs were observed in the PHT and non-PHT groups. Three cases of hepatic decompensation in the PHT group, unrelated to $\mathrm{G} / \mathrm{P}$ according to the investigators, were reported. Conclusion: G/P treatment for 8 or 12 weeks was equally efficacious in HCV patients with features of PHT. Safety outcomes were similar between PHT and non-PHT groups, with G/P treatment well tolerated across groups.

NCTs: NCT03089944, NCT02642432, NCT02738138, NCT02243293, NCT02651194, NCT03235349, NCT02707952, NCT02966795, NCT03069365, NCT03219216. 
Keywords: Glecaprevir; Hepatitis C; Pibrentasvir; Portal Hypertension; Sustained Virologic Response

\section{Key Summary Points}

\section{Why carry out this study?}

Patients with HCV infection and cirrhosis are at increased risk of developing complications such as portal hypertension (PHT).

Direct-acting antivirals, such as glecaprevir/pibrentasvir $(\mathrm{G} / \mathrm{P})$, have been shown to be effective and well tolerated in the majority of patients, but further data are needed in patients with compensated cirrhosis (CC) and evidence of PHT.

This study evaluated the efficacy of 8versus 12 -week $\mathrm{G} / \mathrm{P}$ in treatment-naïve (TN) patients with CC and evidence of PHT and safety in patients with versus those without evidence of PHT.

\section{What was learned from this study?}

Rates of sustained virologic response were high (>97.5\%) for both 8 - and 12-week $\mathrm{G} / \mathrm{P}$ in patients with $\mathrm{CC}$ and evidence of PHT and there were no differences in adverse events (AEs) between patients with or without signs of PHT.

This analysis shows that 8 -week G/P is efficacious and well tolerated in TN patients with CC and evidence of PHT, supporting the simplified treatment of hepatitis $C$ in patients with advanced liver disease.

\section{INTRODUCTION}

Chronic hepatitis $\mathrm{C}$, an infection related to substantial liver disease, has been estimated to affect 58 million individuals worldwide in 2019 [1]. If left untreated, approximately $5-25 \%$ of 
versus 12-week G/P in treatment-naïve $\mathrm{HCV}$ infected patients with $\mathrm{CC}$ and clinical evidence suggestive of PHT prior to treatment, and to assess the safety of patients with evidence of PHT compared with those without.

\section{METHODS}

\section{Study Design and Patient Population}

Data were derived from an ad hoc subgroup analysis of the EXPEDITION-8 study for patients receiving 8 weeks of G/P therapy [6]. For patients receiving 12 weeks of therapy, data were derived from a subgroup analysis of nine (one phase 2 and eight phase 3 studies) pooled studies: EXPEDITION-1 (NCT02642432) [14], EXPEDITION-2 (NCT02738138) [9], SURVEYOR-II (NCT022 43293) [15], EXPEDITION-4 (NCT02651194) [16], VOYAGE-2 (NCT03235349) [10], CERTAIN-1 (NCT02707952) [17], ENDURANCE-5,6 (NCT0296 6795) [8], EXPEDITION-5 (NCT03069365) [18], and EXPEDITION-3 (NCT03219216) [19]. Since this was a post hoc analysis on aggregated, deidentified data, no ethics committee approval was needed. All trial protocols upon which this post hoc analysis was based were approved by the independent ethics committees or institutional review boards at all the trial centers. All the trials were conducted in accordance with Good Clinical Practice Guidelines and the ethical principles of the Declaration of Helsinki, all participants provided written informed consent and parents/guardians provided consent where required, and study participants provided consent where required [6, 8-10, 14-19].

The study designs, patient populations, and outcomes of the trials included in this analysis have been published previously [8-10, 14-19]. In brief, patients were TN, infected with chronic HCV genotypes (GT)1-6, and had CC. Patients categorized as belonging to the PHT groups needed to exhibit at least one of the following features of PHT at baseline (on or prior to Day 1 of $\mathrm{G} / \mathrm{P})$ : FibroScan $\geq 20 \mathrm{kPA}$, platelets $<100 \times 10^{9} / \mathrm{L}$, or a medical history consistent with PHT [defined as the presence of one or more clinical manifestations of PHT (i.e., gastroesophageal varices, ascites, hepatic encephalopathy) described in the patient's medical history at baseline)]. Patients with features indicative of severe PHT or decompensated cirrhosis were excluded from these studies. Laboratory exclusion criteria were: total bilirubin $>3.0 \mathrm{mg} / \mathrm{dL}$, alanine aminotransferase (ALT) or aspartate aminotransferase $($ AST) $>10 \times$ upper limit of normal $(\mathrm{ULN})$, or platelet count $<50,000-60,000$ cells $/ \mathrm{mm}^{3}$ (depending on the specific protocol).

\section{Endpoints and Assessments}

Patient demographics and clinical characteristics were recorded at baseline and included sex, race, age, GT, body mass index (BMI), platelet count, albumin level, transient elastography, Fib-4 scores, Child-Pugh scores, and fibrosis stage. Comorbidities of interest (HIV coinfection, alcohol use, and history of injection drug use) were also reported. Cirrhosis status was determined within each study protocol and was not reassessed for this analysis. CC was defined by liver biopsy [METAVIR fibrosis stage F4 (or equivalent)], transient elastography (Fibroscan ${ }^{\circledR}$, Echosens, France) $\geq 14.6 \mathrm{kPa}$, a FibroTest (BioPredictive, Paris, France) score of $\geq 0.75$, and an aspartate aminotransferase (AST)-toplatelet ratio index (APRI) of $>2$, a Child-Pugh score of 6 or less at screening, and no current or past evidence of Child-Pugh B or C classification or clinical history of liver decompensation. Absence of HCC was confirmed at or within 3 months prior to screening by a negative ultrasound, computed tomography scan, or magnetic resonance imaging [6, 8-10, 14-19].

The primary efficacy endpoint of each of the included studies was the percentage of patients who achieved SVR12 (defined as HCV RNA below the lower limit of quantification 12 weeks after treatment). Additional analyses included the SVR12 rate in patients with GT3. Safety outcomes were also assessed. In all the studies, all treatment-emergent adverse events (AEs), defined as any $\mathrm{AE}$ with an onset date after the first dose of $G / P$ and no more than 30 days after the last G/P dose, were monitored and were either coded using the Medical Dictionary for Regulatory Activities v.23.1 Preferred Terms or 
mapped onto this version for the integrated data analysis [6, 8-10, 14-19]. For this analysis, we were focused on differences in SVR12 between 8 and 12 weeks in PHT patients, as well as on differences in safety between PHT and non-PHT groups.

\section{Statistical Analyses}

All patients who received at least one dose of G/P were included in the intention-to-treat (ITT) analysis. Baseline patient demographics and disease characteristics, efficacy, and safety were all analyzed in the ITT population. Categorical variables were summarized by number and percentage; continuous variables were summarized with descriptive statistics.

\section{RESULTS}

There were 343 patients included in the EXPEDITION-8 study who received 8-weeks of therapy; of those, $60.6 \%(208 / 343)$ patients were identified as patients with clinical evidence suggestive of PHT. In this 8-week group, 43.4\% $(149 / 343)$ patients had a FibroScan $\geq 20 \mathrm{kPa}$, $23.3 \%(80 / 343)$ had platelets $<100 \times 10^{9} / \mathrm{L}$, and $20.4 \%(70 / 343)$ had a medical history consistent with features of PHT. Of a total of 392 patients in the pooled analyses of patients receiving 12 weeks of G/P therapy, $57.1 \%(224 / 392)$ were identified as having clinical evidence suggestive of PHT. In the overall 12-week group, $40.6 \%$ $(159 / 392)$ of patients had a FibroScan $\geq 20 \mathrm{kPa}$, $26.0 \%(102 / 392)$ had platelets $<100 \times 10^{9} / \mathrm{L}$, and $13.5 \%(53 / 392)$ had a medical history consistent with features of PHT. Baseline characteristics are described in Table 1.

\section{Efficacy}

In patients with evidence of PHT, SVR12 was achieved by $97.6 \%(203 / 208)$ who received 8 -week G/P and $98.7 \%(221 / 224)$ in those who received 12 weeks of G/P. Similarly, the group without evidence of PHT patients receiving 8-week G/P achieved an SVR12 rate of $97.8 \%$
$(132 / 135)$, and the 12-week group achieved an SVR12 rate of $97.6 \%(164 / 168)$ (Fig. 1).

In patients with HCV GT3 and evidence of PHT, 97.7\% (42/43) patients receiving 8 weeks of $\mathrm{G} / \mathrm{P}$ and $97.6 \%(41 / 42)$ patients receiving 12 weeks of G/P achieved SVR12. In the HCV GT3 patients who did not have evidence of PHT, $90.0 \%(18 / 20)$ patients in the 8-week group and $94.5 \%(52 / 55)$ in the 12-week group achieved SVR12 (Fig. 1).

In the PHT group, 8 patients did not achieve SVR12, of whom 5 were in the 8-week G/P group: 1 GT3-infected patient experienced relapse, 1 GT1b-infected patient prematurely discontinued treatment prior to week 8 (for reasons other than AEs) and was lost to followup after 17 days of treatment, and 3 patients ( 2 patients infected with GT1a and 1 infected with GT1b) had missing SVR12 data. The remaining 3 patients with PHT who did not achieve SVR12 were in the 12-week G/P group: 1 GT1b-infected patient had missing SVR12 data and 2 (1 GT3infected patient and 1 GT6-infected patient) had on-treatment virologic failure (breakthrough). The HCV GT3 patient experienced on-treatment virologic failure (OTVF) at the treatment-week 8 visit following a period of non-compliance with $\mathrm{G} / \mathrm{P}$ dosing, and the patient with HCV GT6 had OTVF at the treatment-week 12 visit, and had a treatment-emergent NS3 RAS (A156M) and NS5A RAS (T93A) at the time of failure.

In the group without clinical evidence suggestive of PHT, 7 patients did not achieve SVR12, 3 (2 with GT3-infection and 1 with GT1a-infection) in the 8-week group had missing SVR12 data. In the 12-week non-PHT G/P group, 4 patients did not achieve SVR12: 1 GT3infected patient relapsed, 2 GT3-infected patients had missing SVR12 data, and 1 GT1ainfected patient prematurely discontinued the study at 27 days of treatment due to a nonserious AE of diarrhea, considered reasonably likely to be related to $\mathrm{G} / \mathrm{P}$, and relapsed after stopping prematurely. 
Table 1 Baseline demographics and clinical characteristics

\begin{tabular}{|c|c|c|c|c|}
\hline Baseline characteristic & $\begin{array}{l}8 \text { weeks } \\
\text { PHT } \\
n=208 \\
n(\%)\end{array}$ & $\begin{array}{l}8 \text { weeks } \\
\text { non-PHT } \\
n=135 \\
n(\%)\end{array}$ & $\begin{array}{l}12 \text { weeks } \\
\text { PHT } \\
n=224 \\
n(\%)\end{array}$ & $\begin{array}{l}12 \text { weeks } \\
\text { non-PHT } \\
n=168 \\
n(\%)\end{array}$ \\
\hline \multicolumn{5}{|l|}{ Sex } \\
\hline Male & $136(65.4)$ & $81(60.0)$ & $127(56.7)$ & $96(57.1)$ \\
\hline Female & $72(34.6)$ & $54(40.0)$ & $97(43.3)$ & $72(42.9)$ \\
\hline \multicolumn{5}{|l|}{ Race } \\
\hline White & $167(80.3)$ & $118(87.4)$ & $103(46.0)$ & $91(54.2)$ \\
\hline Black & $17(8.2)$ & $11(8.1)$ & $10(4.5)$ & $10(6.0)$ \\
\hline Asian & $22(10.6)$ & $6(4.4)$ & $105(46.9)$ & $66(39.3)$ \\
\hline Other & $2(1.0)$ & 0 & $6(2.7)$ & $1(0.6)$ \\
\hline \multicolumn{5}{|l|}{ HCV GT } \\
\hline $1 \mathrm{a} / 1 \mathrm{~b}$ subtype & $\begin{array}{l}64(30.8) / 75 \\
(36.1)\end{array}$ & $\begin{array}{l}31(23.0) / 61 \\
\quad(45.2)\end{array}$ & $\begin{array}{l}34(15.2) / 84 \\
(37.5)\end{array}$ & $\begin{array}{c}22(13.1) / 42 \\
(25.0)\end{array}$ \\
\hline 2 & $15(7.2)$ & $11(8.1)$ & $47(21.0)$ & $30(17.9)$ \\
\hline 3 & $43(20.7)$ & $20(14.8)$ & $42(18.8)$ & $55(32.7)$ \\
\hline $4-6$ & $11(5.3)$ & $12(8.9)$ & $17(7.6)$ & $19(11.3)$ \\
\hline Age (years) (median, range) & $57(32-84)$ & $59(33-88)$ & $59(28-88)$ & $58(26-87)$ \\
\hline BMI $\left(\mathrm{kg} / \mathrm{m}^{2}\right)$ (median, range) & $26.9(18.4-55.4)$ & $27.9(19.0-45.8)$ & $25.7(17.8-55.4)$ & $26.2(15.2-44.8)$ \\
\hline $\begin{array}{l}\text { Baseline platelets }\left(10^{9} / \mathrm{L}\right)(\text { median, } \\
\text { Q1-Q3) }\end{array}$ & $\begin{array}{l}120.5 \\
\quad(90.0-162.5)\end{array}$ & $\begin{array}{l}180.0 \\
\quad(152.0-222.0)\end{array}$ & $\begin{array}{l}111.0 \\
\quad(87.0-159.5)\end{array}$ & $\begin{array}{l}155.5 \\
\quad(128.0-184.5)\end{array}$ \\
\hline $\begin{array}{l}\text { Baseline albumin (g/L) (median, } \\
\text { Q1-Q3) }\end{array}$ & $42.0(39.0-44.0)$ & $43.0(41.0-45.0)$ & $41.0(38.0-44.0)$ & $42.0(40.0-45.0)$ \\
\hline $\begin{array}{l}\text { Baseline FibroScan (kPa) (median, } \\
\text { Q1-Q3) }\end{array}$ & $25.5(21.3-33.1)^{\mathrm{a}}$ & $16.5(15.3-17.5)^{b}$ & $24.4(20.9-34.6)^{c}$ & $16.5(15.0-17.3)^{\mathrm{d}}$ \\
\hline Baseline FIB-4 (median, Q1-Q3) & $4.2(2.7-6.0)$ & $2.2(1.5-3.4)$ & $4.7(3.0-6.9)$ & $2.9(2.0-4.5)$ \\
\hline \multicolumn{5}{|l|}{ Baseline Child-Pugh Score } \\
\hline 5 & $181(87.0)$ & $126(93.3)$ & $193(86.5)$ & $160(95.2)$ \\
\hline 6 & $25(12.0)$ & $8(5.9)$ & $27(12.1)$ & $7(4.2)$ \\
\hline$>6$ & $2(1.0)$ & $1(0.7)$ & $3(1.3)$ & $1(0.6)$ \\
\hline Missing & 0 & 0 & 1 & 0 \\
\hline \multicolumn{5}{|l|}{ Baseline fibrosis stage } \\
\hline F0-F1 & 0 & 0 & 0 & 0 \\
\hline $\mathrm{F} 2$ & 0 & 0 & 0 & $1(0.6)$ \\
\hline
\end{tabular}


Table 1 continued

\begin{tabular}{|c|c|c|c|c|}
\hline Baseline characteristic & $\begin{array}{l}8 \text { weeks } \\
\text { PHT } \\
n=208 \\
n(\%)\end{array}$ & $\begin{array}{l}8 \text { weeks } \\
\text { non-PHT } \\
n=135 \\
n(\%)\end{array}$ & $\begin{array}{l}12 \text { weeks } \\
\text { PHT } \\
n=224 \\
n(\%)\end{array}$ & $\begin{array}{l}12 \text { weeks } \\
\text { non-PHT } \\
n=168 \\
n(\%)\end{array}$ \\
\hline $\mathrm{F} 3$ & 0 & 0 & $1(0.5)$ & $2(1.2)$ \\
\hline $\mathrm{F} 4$ & $208(100)$ & $135(100)$ & $212(99.5)$ & $159(98.1)$ \\
\hline Missing & 0 & 0 & 11 & 6 \\
\hline Presence of HIV co-infection & 0 & 0 & $5(2.2)$ & $9(5.4)$ \\
\hline Alcohol use (current) & $40(19.2)$ & $30(22.2)$ & $40(17.9)$ & $36(21.4)$ \\
\hline History of injection drug use $\mathrm{e}^{\mathrm{e}}$ & $3(1.4)$ & $1(0.7)$ & $7(3.1)$ & $5(3.0)$ \\
\hline
\end{tabular}

Based on an ITT analysis

$B M I$ body mass index, $F I B-4$ Fibrosis-4, GT genotype, $H C V$ hepatitis $\mathrm{C}$ virus, $H I V$ human immunodeficiency virus, $I T T$ intention-to-treat, $P H T$ portal hypertension, $Q$ quartile

${ }^{\mathrm{a}} \mathrm{n}=178$

$\mathrm{b}_{n}=117$

${ }^{\mathrm{c}} n=188$

$\mathrm{d}_{n}=101$

${ }^{\mathrm{e}}$ Within the last 12 months

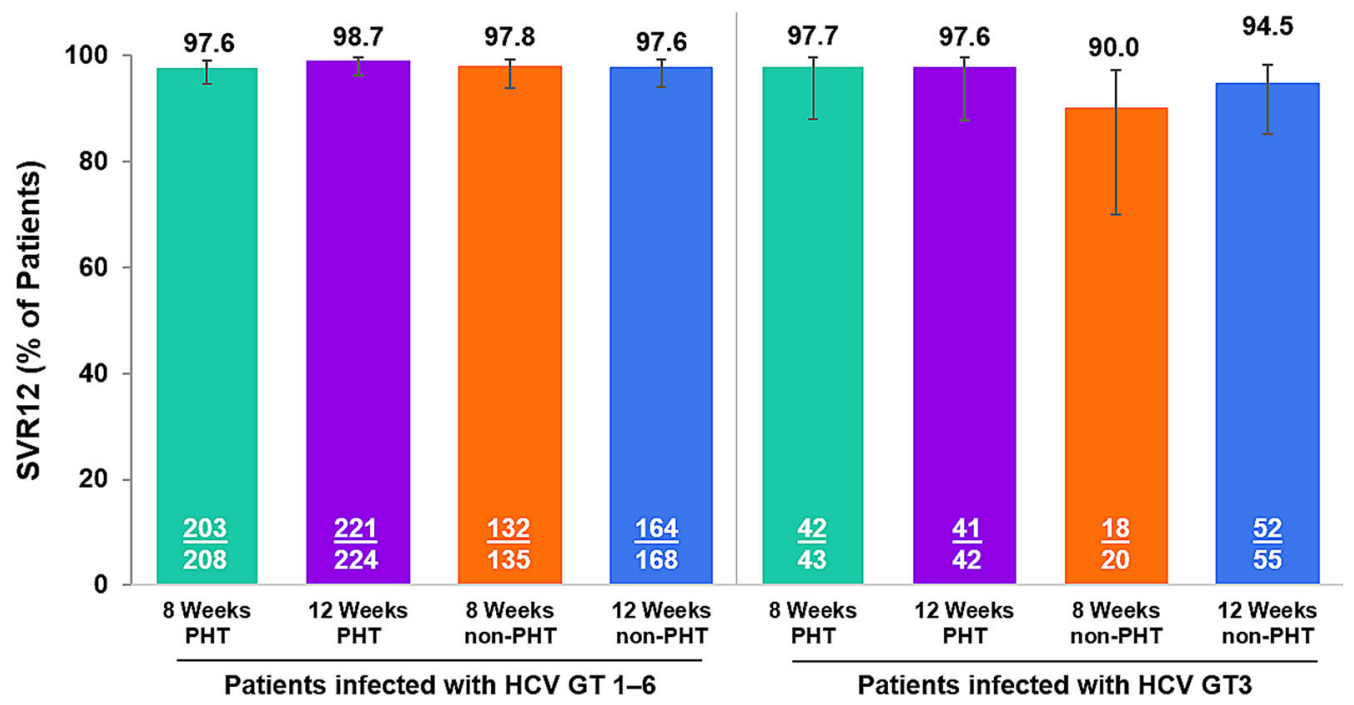

Fig. 1 Percentage of patients who achieved SVR12 receiving 8 or 12 weeks of G/P therapy (ITT analysis); \% SVR12 $\pm 95 \%$ confidence interval. $G / P$ glecaprevir/pibrentasvir, ITT

\section{Safety}

Overall, 28.5\% of patients with evidence of PHT and $28.1 \%$ of patients without PHT experienced intention-to-treat, PHT portal hypertension, SVR 12 sustained virologic response at post-treatment week 12

an AE possibly related to $G / P$, as assessed by an investigator. In patients on 8 weeks of $G / P$ therapy, this was $25.5 \%$ of patients with PHT and $25.9 \%$ of patients without clinical evidence 
Table 2 Adverse events

\begin{tabular}{|c|c|c|c|c|c|c|}
\hline Event & $\begin{array}{l}\text { PHT } \\
\text { overall } \\
(n=432) \\
n(\%)\end{array}$ & $\begin{array}{l}\text { Non-PHT } \\
\text { overall } \\
(n=303) \\
n(\%)\end{array}$ & $\begin{array}{l}8 \text { weeks } \\
\text { PHT } \\
n=208 \\
n(\%)\end{array}$ & $\begin{array}{l}8 \text { weeks } \\
\text { non-PHT } \\
n=135 \\
n(\%)\end{array}$ & $\begin{array}{l}12 \\
\text { weeks } \\
\text { PHT } \\
n=224 \\
n(\%)\end{array}$ & $\begin{array}{l}12 \text { weeks } \\
\text { non-PHT } \\
n=168 \\
n(\%)\end{array}$ \\
\hline Any AE & $224(51.9)$ & $180(59.4)$ & $94(45.2)$ & $64(47.4)$ & $\begin{array}{l}150 \\
(67.0)\end{array}$ & $116(69.0)$ \\
\hline $\begin{array}{l}\text { Any AE with reasonable possibility of } \\
\text { being related to } G / \mathrm{P}^{\mathrm{a}}\end{array}$ & $123(28.5)$ & $85(28.1)$ & $53(25.5)$ & $35(25.9)$ & $\begin{array}{l}70 \\
(31.3)\end{array}$ & $50(29.8)$ \\
\hline \multicolumn{7}{|l|}{ AEs related to $G / P$ in $\geq 5 \%$ of patients } \\
\hline Fatigue & $30(6.9)$ & $24(7.9)$ & $15(7.2)$ & $11(8.1)$ & $15(6.7)$ & $13(7.7)$ \\
\hline Pruritus & $23(5.3)$ & $24(7.9)$ & $12(5.8)$ & $13(9.6)$ & $11(4.9)$ & $11(6.5)$ \\
\hline Headache & $27(6.3)$ & $18(5.9)$ & $14(6.7)$ & $7(5.2)$ & $13(5.8)$ & $11(6.5)$ \\
\hline Nausea & $22(5.1)$ & $12(4.0)$ & $9(4.3)$ & $4(3.0)$ & $13(5.8)$ & $8(4.8)$ \\
\hline AEs of hepatic decompensation ${ }^{b, c}$ & $3(0.7)$ & 0 & $1(0.5)$ & 0 & $2(0.9)$ & 0 \\
\hline Any serious $\mathrm{AE}$ & $16(3.7)$ & $14(4.6)$ & $2(1.0)$ & $4(3.0)$ & $14(6.3)$ & $10(6.0)$ \\
\hline Any G/P-related serious AE & $1(0.2)$ & 0 & 0 & 0 & $1(0.4)$ & 0 \\
\hline AE leading to drug discontinuation & $2(0.5)$ & $1(0.3)$ & 0 & 0 & $2(0.9)$ & $1(0.6)$ \\
\hline $\begin{array}{l}\text { Any G/P-related AE leading to drug } \\
\text { discontinuation }\end{array}$ & $1(0.2)$ & $1(0.3)$ & 0 & 0 & $1(0.4)$ & $1(0.6)$ \\
\hline Any AE leading to interruption of $\mathrm{G} / \mathrm{P}$ & $3(0.7)^{\mathrm{d}}$ & $3(1.0)^{\mathrm{e}}$ & $1(0.5)^{\mathrm{d}}$ & 0 & $2(0.9)^{\mathrm{d}}$ & $3(1.8)^{\mathrm{e}}$ \\
\hline Any AE leading to death & $1(0.2)^{\mathrm{f}}$ & 0 & 0 & 0 & $1(0.4)^{f}$ & 0 \\
\hline
\end{tabular}

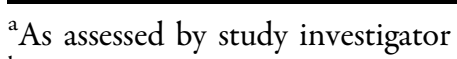

${ }^{b}$ Deemed unrelated to G/P by study investigators

${ }^{\mathrm{c}}$ One nonserious case of ascites was reported in the 8-week PHT group; 1 nonserious case of ascites and 1 serious case of esophageal variceal bleeding were reported in the 12-week PHT group

${ }^{\mathrm{d}}$ Patient(s) achieved SVR12 despite interruption

${ }^{\mathrm{e}}$ All but one patient achieved SVR12

$\mathrm{f}$ 41-year-old Asian male died of left cerebral hemorrhage deemed unrelated to $\mathrm{G} / \mathrm{P}$ by the study investigator $A E$ adverse event, $G / P$ glecaprevir/pibrentasvir, ITT intention-to-treat, $P H T$ portal hypertension

of PHT, and in patients on 12 weeks of G/P therapy, this was $31.3 \%$ of patients with PHT and $29.8 \%$ of patients without evidence of PHT (Table 2). The most common AEs related to G/P $(\geq 5 \%$ in any group) were fatigue, pruritus, headache, and nausea.

In patients with PHT, three cases of hepatic decompensation were reported overall (8- and 12-week groups): one nonserious case of ascites was reported in the 8-week group; one nonserious case of ascites, and one serious case of esophageal variceal bleeding were reported in the 12-week group. None of these events of hepatic decompensation were deemed related to $\mathrm{G} / \mathrm{P}$ by the investigators, nor led to the discontinuation of $\mathrm{G} / \mathrm{P}$ therapy. All events of hepatic decompensation, with the exception of the one case of moderate ascites occurring in the 8-week group, resolved. Moderate ascites 
was present at baseline, where the patient had a Child-Pugh B score of 7 . This rendered the patient's study enrollment a protocol violation. By the end of the study, ascites was ongoing and deemed unrelated to G/P. The patient completed treatment and achieved SVR12, as did the other two patients with hepatic decompensation. There were no AEs related to hepatic decompensation reported in patients without PHT, irrespective of treatment duration.

\section{DISCUSSION}

The data in this paper build on those in large real-world cohorts across wide ranging populations covering those with cirrhosis, pediatric patients, and those with GT3 infection, further demonstrating the breadth of applicability for the regimen without compromising safety/efficacy [11, 20-25]. In this analysis, the proportion of patients with clinical evidence suggestive of PHT at baseline was similar between groups treated with 8 and 12 weeks of G/P, suggesting that the patient population in EXPEDITION-8 was similar to those in previous clinical trials of 12-week G/P. Importantly, the analysis also showed that SVR12 rates were similarly high $(\geq 97.6 \%)$ for patients with evidence of PHT, regardless of whether they were treated with 8 or 12 weeks of G/P. In addition, there were no differences in SVR12 rates between patients with and without evidence of PHT ( $\geq 97.6 \%$ ). The efficacy data reported in this analysis are comparable to data from the clinical trials of G/P in patients both with and without CC. In an integrated analysis of TN patients with HCV GT1-6, treated with 8 weeks of G/P, ITT SVR rates for patients with CC were $97.7 \%$ and in patients without cirrhosis were 97.6\% [7]. These high SVR rates are especially encouraging, since SVR by DAAs has been shown to reduce the risk of many extrahepatic manifestations, including acute coronary syndrome, cardiovascular disease, insulin resistance, and type 2 diabetes, as well as reducing the risk of developing cryoglobulinemic vasculitis and B-cell non-Hodgkin's lymphomas [25].

Similarly, high rates of SVR12 were seen for the subset of patients infected with HCV GT3, with results being comparable for those treated with 8-week and 12-week G/P, regardless of whether they showed clinical evidence suggestive of PHT at the baseline.

G/P was well tolerated across all treatment groups, AEs were comparable between patients with and without evidence of PHT, and no new or unexpected safety signals were observed. Across all subgroups and consistent with the known safety profile of $\mathrm{G} / \mathrm{P}$, the most commonly reported AEs assessed with a reasonable possibility of being related to $G / P$ by the investigator (headache, pruritus, fatigue, and nausea) were similar for 8 - and 12 -week G/P [6-11]. Similarly, rates of serious AEs $(\sim 4 \%)$ and AEs with a reasonable possibility of being related to $\mathrm{G} / \mathrm{P}(\sim 28 \%)$ were similar when comparing subgroups with evidence of PHT at baseline to those without and were consistent with previously reported results [6-11]. Cases of hepatic decompensation-related AEs were rare, and, while only seen in the PHT subgroups, they were deemed to be unrelated to $\mathrm{G} / \mathrm{P}$ by the study investigators; none led to treatment discontinuation, serious complications, or liver failure/death, and all achieved SVR12 [7].

The data from this analysis provide reassurance around both the safety of G/P in patients with PHT and the efficacy of 8 versus 12 weeks of treatment with $\mathrm{G} / \mathrm{P}$ in this population. It is important to note that patients included in this analysis had evidence of PHT but no current decompensation events. Additionally, patients with severe PHT (platelets $<50,000-60,000$ cells $/ \mathrm{mm}^{3}$ ), patients who had decompensated cirrhosis (Child-Pugh scores $>7$ ) or ALT or AST $>10 \times$ ULN were excluded from the trials included in this analysis [6, 8-10, 14-19]. The generalizability of the data presented here obtained from clinical trials may need to be considered in relation to settings where PHT is assessed through alternative methods of testing.

The diagnosis of advanced liver disease and cirrhosis is not always straightforward, and it is important to acknowledge that these are a series of progressively worsening disease states, which can be difficult to define [26]. Most guidelines, including those provided by EASL, recommend the use of non-invasive tests over liver biopsy to determine the extent of fibrosis/cirrhosis in 
chronic hepatitis C. EASL guidelines also provide recommendations on a number of the tests that can be used, including transient elastography $(\mathrm{F} 4 \geq 13 \mathrm{kPa})$, FibroTest $(\mathrm{F} 4 \geq 0.74)$, and aminotransferase to platelet ratio index (APRI; F4 2 2) [5]. Similarly, PHT has historically been difficult to define, and definitions continue to evolve. Current recommendations from EASL suggest the use of Baveno VI criteria for identifying patients with CC (Child-Pugh A) and features of PHT, specifically, liver stiffness $>20 \mathrm{kPa}$ with a platelet count $<150 \times 10^{9} /$ $\mathrm{L}[4,5]$. The criteria used in this analysis to define both patients with CC and those with clinical evidence suggestive of PHT meet or exceed the criteria suggested by EASL, affording confidence that patients included in this analysis are likely an accurate representation of realworld compensated cirrhotic and PHT patient populations. This provides further reassurance for clinicians utilizing 8-week G/P therapy in TN HCV-infected patients with evidence of PHT without overt decompensation.

There are some limitations to this analysis that are inherent in its design. For example, it is an ad hoc analysis, meaning that there is less control of patient inclusion, which can lead to biased data. A further limitation is that these results cannot be generalized to patients with severe PHT (platelet count $<50,000 \mathrm{cell} / \mathrm{mm}^{3}$ ), as these patients were excluded from the studies upon which this post hoc analysis is based. In addition, meaningful analysis of subgroups with varying severity of PHT cannot be performed, as there is a relatively low number of patients with platelet count $<100,000$ cell/ $\mathrm{mm}^{3}$. The very small numbers of non-SVR and decompensation AEs identified in this study preclude the identification of risk factors for these negative outcomes. Finally, due to the exclusion of those with more severe PHT, it was not possible to explore the impact of different platelet levels in a meaningful way.

Despite the availability of DAAs that require minimal pre-treatment testing, determining the presence of advanced liver disease and cirrhosis remains an important step, particularly in terms of post-treatment monitoring for $\operatorname{HCC}[5,26]$. The results from the current analysis suggest that 8-week G/P is efficacious and well tolerated in patients with clinical evidence suggestive of PHT but without decompensated liver disease, further supporting the simplification of treatment for patients with HCV infection, even in those with more advanced liver disease.

\section{CONCLUSIONS}

This analysis shows that 8-week treatment with $\mathrm{G} / \mathrm{P}$ is both efficacious and well tolerated in $\mathrm{TN}$ patients with CC and evidence of PHT. G/P treatment for 8 or 12 weeks was equally efficacious, with rates of sustained virologic response $>97.5 \%$ in HCV patients with CC and features of PHT. Safety outcomes were similar between PHT and non-PHT groups, with G/P treatment well tolerated across groups. Thus, our results support the simplified treatment of hepatitis $\mathrm{C}$ with short-duration G/P therapy in patients with advanced liver disease.

\section{ACKNOWLEDGEMENTS}

The authors would like to express their gratitude to the patients who participated in the previous studies (NCT03089944, NCT02642432, NCT02738138, NCT02243293, NCT02651194, NCT03235349, NCT02707952, NCT02966795, NCT03069365, NCT03219216), and their families, as well as the study investigators and coordinators of the study. Glecaprevir was identified by AbbVie and Enanta. Medical writing support was provided by Sneh Mody, PharmD, MBA, BCCCP of AbbVie, and Jeannine Delwiche, $\mathrm{PhD}$, and Laura Whiteley, $\mathrm{PhD}$, of Fishawack Health and funded by AbbVie.

Funding. The design, study conduct, analysis, and financial support of the study (NCTs: NCT03089944, NCT02642432, NCT02738138, NCT02243293, NCT02651194, NCT03235349, NCT02707952, NCT02966795, NCT03069365, NCT03219216) was provided by AbbVie. AbbVie participated in the study design, research, analysis, data collection, interpretation of data, review, and approval of the publication. All authors had access to all relevant 
data and participated in writing, review, and approval of this manuscript. No honoraria or payments were made for authorship. Abbvie funded the journal's Rapid Service Fees.

Authorship. All named authors meet the International Committee of Medical Journal Editors (ICMJE) criteria for authorship for this article, take responsibility for the integrity of the work as a whole, and have given their approval for this version to be published.

Author Contributions. Drs. RS Brown, Jr., MA Collins, SI Strasser, A Emmett, AS Topp, M Burroughs, R Ferreira, and JJ Feld all participated in the concept, design, analysis, draft and review of this manuscript.

Disclosures. Brown RS: Research support: AbbVie, Gilead, Enanta Intercept Advisor: AbbVie, Gilead, Intercept, and Mallinckrodt. Strasser SI: Advisory Board/Speaker: AbbVie, Gilead, Bristol-Myers Squibb, MSD, Roche, Eisai, AstraZeneca, Ipsen, Dr Falk Pharma, and CSL Behring. Feld JJ: Research support/consultant: AbbVie, Antios, Arbutus, Eiger, Enanta, Finch, Gilead, GSK, Janssen, and Roche. Collins MA, Emmett A, Topp A, Burroughs M, and Ferreira R: Employees of AbbVie Inc. and may hold stock or options.

Compliance with Ethics Guidelines. No ethics committee approval was needed for this manuscript as it was a post-hoc analysis on aggregated, de-identified data. The original studies on which this subanalysis was based upon were all approved by ethics committees.

Data Availability. AbbVie is committed to responsible data sharing regarding the clinical trials we sponsor. This includes access to anonymized, individual and trial-level data (analysis data sets), as well as other information (e.g., protocols and Clinical Study Reports), as long as the trials are not part of an ongoing or planned regulatory submission. This includes requests for clinical trial data for unlicensed products and indications. These clinical trial data can be requested by any qualified researchers who engage in rigorous, independent scientific research, and will be provided following review and approval of a research proposal and Statistical Analysis Plan (SAP) and execution of a Data Sharing Agreement (DSA). Data requests can be submitted at any time and the data will be accessible for 12 months, with possible extensions considered. For more information on the process, or to submit a request, visit the following link: https://www.abbvie.com/ourscience/clinical-trials/clinical-trials-data-andinformation-sharing/data-and-informationsharing-with-qualified-researchers.html.

Open Access. This article is licensed under a Creative Commons Attribution-NonCommercial 4.0 International License, which permits any non-commercial use, sharing, adaptation, distribution and reproduction in any medium or format, as long as you give appropriate credit to the original author(s) and the source, provide a link to the Creative Commons licence, and indicate if changes were made. The images or other third party material in this article are included in the article's Creative Commons licence, unless indicated otherwise in a credit line to the material. If material is not included in the article's Creative Commons licence and your intended use is not permitted by statutory regulation or exceeds the permitted use, you will need to obtain permission directly from the copyright holder. To view a copy of this licence, visit http:// creativecommons.org/licenses/by-nc/4.0/.

\section{REFERENCES}

1. World Health Organization. Global progress report on hiv, viral hepatitis and sexually transmitted infections. Geneva: WHO; 2021.

2. Akinyemiju T, Abera S, Ahmed M, et al. The burden of primary liver cancer and underlying etiologies from 1990 to 2015 at the global, regional, and national level: results from the global burden of disease study 2015. JAMA Oncol. 2017;3(12): 1683-91.

3. Liu Z, Jiang Y, Yuan H, et al. The trends in incidence of primary liver cancer caused by specific etiologies: results from the global burden of disease study 2016 
and implications for liver cancer prevention. J Hepatol. 2019;70(4):674-83.

4. de Franchis R. Expanding consensus in portal hypertension: report of the baveno vi consensus workshop: stratifying risk and individualizing care for portal hypertension. J Hepatol. 2015;63(3): 743-52.

5. European Association for the Study of the Liver. Easl recommendations on treatment of hepatitis c: final update of the series. J Hepatol. 2020;73(5): 1170-218.

6. Brown RS Jr, Buti M, Rodrigues L, et al. Glecaprevir/ pibrentasvir for 8 weeks in treatment-naive patients with chronic hcv genotypes 1-6 and compensated cirrhosis: the expedition-8 trial. J Hepatol. 2020;72(3):441-9.

7. Zuckerman E, Gutierrez JA, Dylla DE, et al. Eight weeks of treatment with glecaprevir/pibrentasvir is safe and efficacious in an integrated analysis of treatment-naïve patients with hepatitis $\mathrm{c}$ virus infection. Clin Gastroenterol Hepatol. 2020;18(11): 2544-53.e6.

8. Asselah T, Lee SS, Yao BB, et al. Efficacy and safety of glecaprevir/pibrentasvir in patients with chronic hepatitis c virus genotype 5 or 6 infection (endurance-5,6): an open-label, multicentre, phase $3 \mathrm{~b}$ trial. Lancet Gastroenterol Hepatol. 2019;4(1): 45-51.

9. Rockstroh JK, Lacombe K, Viani RM, et al. Efficacy and safety of glecaprevir/pibrentasvir in patients coinfected with hepatitis $\mathrm{c}$ virus and human immunodeficiency virus type 1: the expedition-2 study. Clin Infect Dis. 2018;67(7):1010-7.

10. Wei L, Wang G, Alami NN, et al. Glecaprevir-pibrentasvir to treat chronic hepatitis c virus infection in asia: Two multicentre, phase 3 studies-a randomised, double-blind study (voyage-1) and an open-label, single-arm study (voyage-2). Lancet Gastroenterol Hepatol. 2020;5(9):839-49.

11. Lampertico P, Mauss S, Persico M, et al. Real-world clinical practice use of 8-week glecaprevir/pibrentasvir in treatment-naïve patients with compensated cirrhosis. Adv Ther. 2020;37(9):4033-42.

12. Abbvie. Maviret [Summary of Product Characteristics]. 2021 [cited 2021 September 10]. Available from: https://www.ema.europa.eu/en/documents/ product-information/maviret-epar-productinformation_en.pdf.

13. AbbVie. Mavyret. North Chicago, IL; 2021.

14. Forns X, Lee SS, Valdes J, et al. Glecaprevir plus pibrentasvir for chronic hepatitis c virus genotype
$1,2,4,5$, or 6 infection in adults with compensated cirrhosis (expedition-1): A single-arm, open-label, multicentre phase 3 trial. Lancet Infect Dis. 2017;17(10):1062-8.

15. Wyles D, Poordad F, Wang S, et al. Glecaprevir/pibrentasvir for hepatitis c virus genotype 3 patients with cirrhosis and/or prior treatment experience: a partially randomized phase 3 clinical trial. Hepatology. 2018;67(2):514-23.

16. Gane E, Lawitz E, Pugatch D, et al. Glecaprevir and pibrentasvir in patients with hcv and severe renal impairment. N Engl J Med. 2017;377(15):1448-55.

17. Toyoda H, Chayama K, Suzuki F, et al. Efficacy and safety of glecaprevir/pibrentasvir in japanese patients with chronic genotype 2 hepatitis c virus infection. Hepatology. 2018;67(2):505-13.

18. Lawitz E, Flisiak R, Abunimeh M, et al. Efficacy and safety of glecaprevir/pibrentasvir in renally impaired patients with chronic hcv infection. Liver Int. 2020;40(5):1032-41.

19. Peribanez-Gonzalez M, Cheinquer H, Rodrigues L, et al. Efficacy and safety of glecaprevir/pibrentasvir in treatment-naive adults with chronic hepatitis c virus genotypes 1-6 in brazil. Ann Hepatol. 2021;20:100257.

20. Forns X, Feld JJ, Dylla DE, et al. Safety of patients with hepatitis $c$ virus treated with glecaprevir/pibrentasvir from clinical trials and real-world cohorts. Adv Ther. 2021;38(6):3409-26.

21. Jonas MM, Rhee S, Kelly DA, et al. Pharmacokinetics, safety, and efficacy of glecaprevir/pibrentasvir in children with chronic hcv: Part 2 of the dora study. Hepatology. 2021;74(1):19-27.

22. Klinker H, Naumann U, Rossle M, et al. Glecaprevir/ pibrentasvir for 8 weeks in patients with compensated cirrhosis: safety and effectiveness data from the german hepatitis c-registry. Liver Int. 2021;41(7):1518-22.

23. Su PY, Chen YY, Lai JH, et al. Real-world experience of chronic hepatitis c-related compensated liver cirrhosis treated with glecaprevir/pibrentasvir: a multicenter retrospective study. J Clin Med. 2021;10(22):5236.

24. Wedemeyer H, Erren P, Naumann U, et al. Glecaprevir/pibrentasvir is safe and effective in hepatitis c patients with cirrhosis: real-world data from the german hepatitis c-registry. Liver Int. 2021;41(5):949-55.

25. Zarebska-Michaluk D, Jaroszewicz J, ParfieniukKowerda A, et al. Effectiveness and safety of pangenotypic regimens in the most difficult to treat 
population of genotype $3 \mathrm{hcv}$ infected cirrhotics. J Clin Med. 2021;10(15):3280.

26. Ferenci P. Are all cirrhotic patients equal? J Hepatol. 2020;72(3):389-90.

\section{Publisher's Note}

Springer Nature remains neutral with regard to jurisdictional claims in published maps and institutional affiliations. 